resulted in elimination of vent port obstruction, such that the pump could again function electrically. This averted the need for surgical replacement of the pump. Through this experience, we propose an algorithm, which might help caregivers with this difficult situation (Figure 2).

\section{Reference}

1. Rose EA, Gelijns AC, Moskowitz AJ, Heitjan DF, Stevenson LW, Dembitsky W, et al. Long-term mechanical left ventricular assistance for end-stage heart failure. N Engl J Med. 2001;345:1435-43.

\title{
Surgical management of bilateral multiple invasive pulmonary aspergillosis
}

\author{
Karin M. Dunst, MD, and Ludwig C. Mueller, MD, Innsbruck, Austria
}

$\mathrm{T}$ here are 3 clinical types of pulmonary aspergillosis. The allergic and saprophytic forms can be encountered in immunocompetent patients, whereas invasive pulmonary aspergillosis (IPA) is a life-threatening opportunistic infectious complication that develops in the immunocompromised host as a consequence of impaired defense function. ${ }^{1}$ IPA represents a major source of morbidity and mortality in the neutropenic patient. The clinical symptoms are sometimes masked, especially in the setting of a malignant neoplastic disorder; however, as a rule, there is productive cough and hemoptysis up to life-threatening bleeding. Standard therapy consists of amphotericin B, itraconazole, or both ${ }^{2}$; however, new antifungal agents, such as voriconazole ${ }^{3}$ or caspofungin, ${ }^{4}$ might yield superior therapeutic results. Surgical removal of infected lung tissue is considered as a therapy of last resort. ${ }^{5}$

\section{Clinical Summary}

A 63-year-old woman received high-dose cortisone therapy because of a local seroma complication after surgical removal of a thoracic ependymoma. One month later, she had a septic shocklike syndrome with acute respiratory insufficiency, requiring orotracheal intubation with 100\% oxygen and positive end-expiratory pressure $\left(10 \mathrm{~cm} \mathrm{H}_{2} \mathrm{O}\right)$. This management led to a satisfactory arterial oxygen saturation level (88\%). Bronchoalveolar lavage and sputum cultures revealed Aspergillus fumigatus, and antifungal therapy with amphotericin B was initiated. Thoracic computed tomography showed multiple focal cavitary mycotic lesions, with a cavitary pattern in both of the upper lobes and dense infiltrates in

\footnotetext{
From the Department of Cardiac Surgery, Leopold-Franzens University, Innsbruck, Austria.

Received for publication Jan 6, 2004; revised Jan 31, 2004; accepted for publication Feb 4, 2004.

Address for reprints: Karin M. Dunst, MD, Department of Cardiac Surgery, University Hospital Innsbruck, Anichstrasse 35, 6020 Innsbruck, Austria (E-mail: Karin.dunst@uibk.ac.at).

J Thorac Cardiovasc Surg 2004;128:621-2

$0022-5223 / \$ 30.00$

Copyright (C) 2004 by The American Association for Thoracic Surgery doi:10.1016/j.jtcvs.2004.02.015
}

the lower lobes (Figure 1). Surgical drainage of the largest cavities was undertaken because of failure of conservative management. After 8 weeks of antimycotic treatment, we decided to perform surgical resection. By that

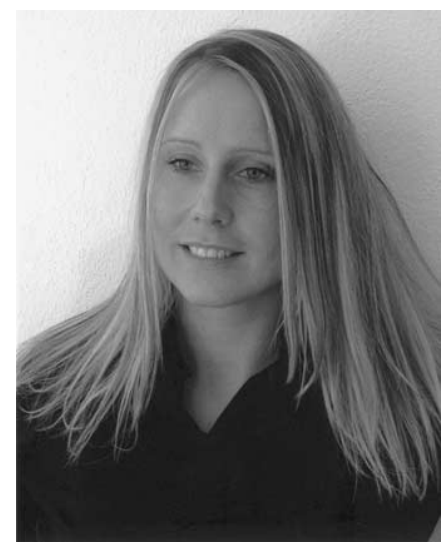

Dr Dunst time, the patient was ventilated through a tracheostoma, with intermittent positive airway pressure. In a first operation, a right upper lobectomy was undertaken (Figure 2). Because of the patient's critical condition, a left upper lobe segmental resection was carried out 4 days later. Furthermore, inflammatory infiltrates in both lungs were omitted from surgical resection. The postoperative course was uneventful, and the patient was discharged from the hospital after 10 days for rehabilitation. At 18 months' followup, the patient has slight exertional dyspnea, and the chest radio-

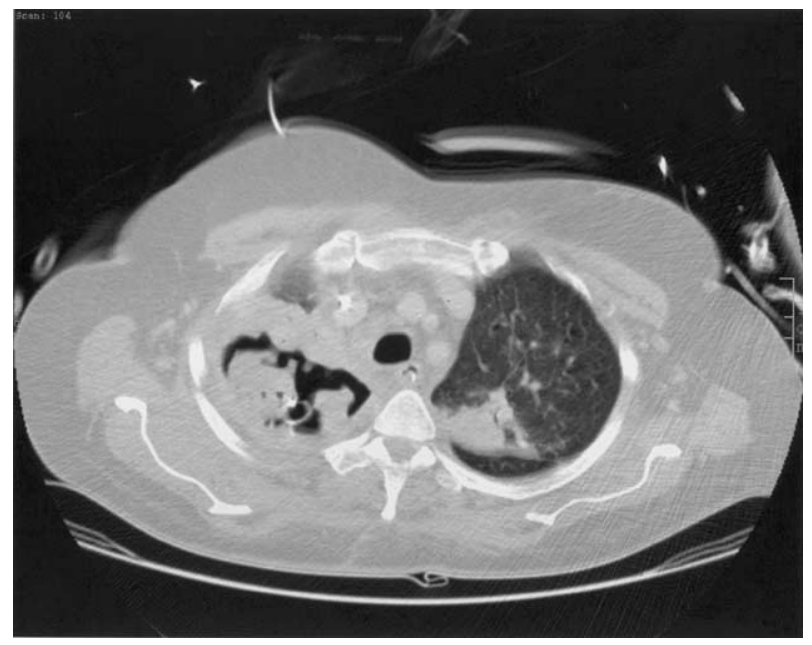

Figure 1. Thoracic computed tomogram showing multiple cavitary lesions with surgical drainage in the right upper lobe. Additionally, there is a consolidated lesion in the left upper lobe, with occasional air trapping. 


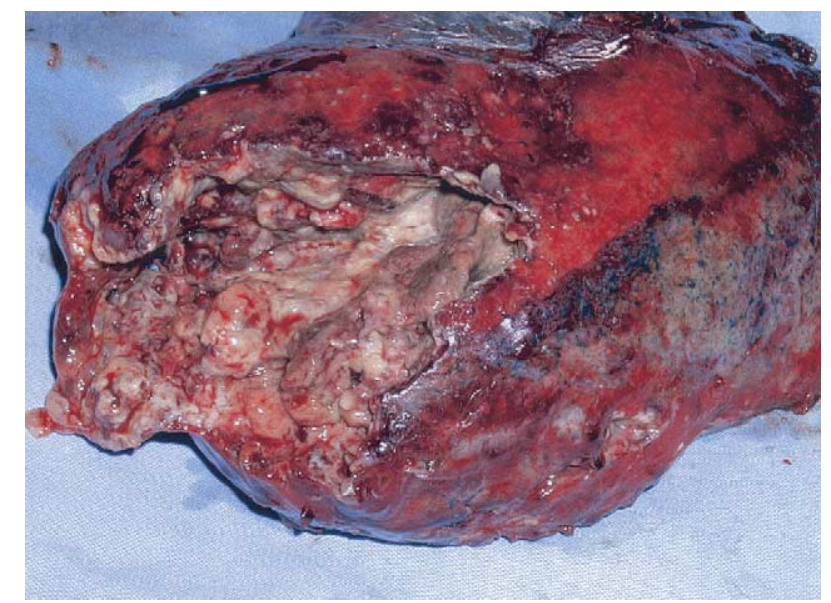

Figure 2. Intraoperative appearance of the resected right upper pulmonary lobe.

graph revealed complete resolution of all fungal infiltrates (Figure $3)$.

\section{Discussion}

This case of IPA demonstrates that even with bilateral multiple involvement, combined conservative and surgical therapy can lead to a favorable outcome. After prolonged conservative therapy without any marked improvement of the pulmonary lesions, a surgical resection has to be indicated. Surgical drainage of mycotic lesions alone will not be sufficient and is associated with considerable morbidity. The target of any surgical intervention must be a cavitary lesion that should be operated on in 1 or 2 sessions. Inflammatory infiltrates usually cannot be treated with resection because of the extent of spreading and should therefore be treated with antimycotic drugs, such as voriconazole and caspofungin. We conclude that resection of affected lung tissue in IPA is feasible with acceptable morbidity and mortality and leads to a markedly improved outcome.

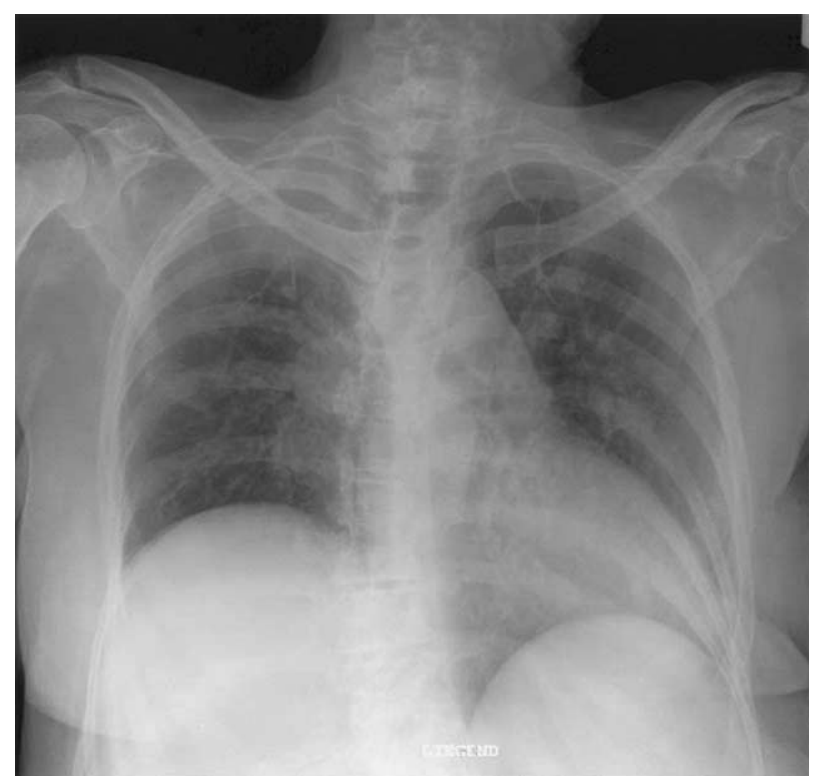

Figure 3. Postoperative chest radiograph at 18 months' follow-up.

\section{References}

1. Soubani AO, Chandrasekar PH. The clinical spectrum of pulmonary aspergillosis. Chest. 2002;121:1988-99.

2. Reichenberger F, Habicht JM, Grathwohl A, Tamm M. Diagnosis and treatment of invasive pulmonary aspergillosis in neutropenic patients. Eur Respir J. 2002;19:743-55.

3. Herbrecht R, Denning DW, Patterson TF, Bennett JE, Greene RE, Oestmann JW, et al. Voriconazole versus amphotericin B for primary therapy of invasive aspergillosis. N Engl J Med. 2002;347:408-15.

4. Taccone FS, Marechal R, Meuleman N, Aoun M. Caspofungin salvage therapy in a neutropenic patient with probable invasive aspergillosis: a case report. Support Care Cancer. 2003;11:742-4.

5. Matt P, Bernet F, Habicht J, Gambazzi F, Passweg J, Grathwohl A, et al. Short- and long-term outcome after lung resection for invasive pulmonary aspergillosis. Thorac Cardiovasc Surg. 2003;51:221-5. 